\title{
Schwinger-Dyson approach to color superconductivity in dense QCD
}

\author{
Deog Ki Hong \\ Department of Physics, Pusan National University, Pusan 609-735, Korea* \\ Physics Department, Boston University, Boston, MA 02215, USA \\ V.A. Miransky \\ Bogolyubov Institute for Theoretical Physics, 252143, Kiev, Ukraine \\ Department of Applied Mathematics, University of Western Ontario, London, Ontario N6A 5B9, Canada \\ I.A. Shovkovy ${ }^{\dagger}$ and L.C.R. Wijewardhana \\ Physics Department, University of Cincinnati, Cincinnati, Ohio 45221-0011
}

(September 17, 1999)

\begin{abstract}
The problem of color superconductivity in dense QCD is reconsidered in the improved rainbow approximation to the Schwinger-Dyson equation. The effect of the unscreened magnetic modes of gluons on the value of the color condensate is studied. In particular, it is shown that, at sufficiently large values of the chemical potential, these modes lead to the enhancement of the superconducting order parameter. The interplay between the instanton induced interaction and the one-gluon induced one in color superconductivity is discussed.
\end{abstract}

11.15.Ex, 12.38.Aw, 12.38.-t, 26.60.+c

\section{INTRODUCTION}

Quantum chromodynamics (QCD) has been remarkably successful in describing the interactions of quarks and gluons at short distance scales. In this distance region, QCD is a perturbative theory due to asymptotic freedom [1]. At large distances, on the other hand, the effective running coupling of QCD becomes strong and non-perturbative approaches, like sum rule methods [2], lattice computer simulations [3], instanton computations [4], and SchwingerDyson equations for Green's functions [5] should be utilized to analyze the theory.

There exist, however, some extreme conditions where QCD is yet to be tested. An example of such an extreme condition is cold quark matter at high density. There have been attempts to investigate dense QCD using perturbation theory [6], instanton calculations [7 9] and lattice simulations [10]. Such dense matter may exist in the interior of neutron or the so-called strange stars [11], with baryon number densities exceeding a few times the normal nuclear density $n_{0} \simeq 0.17 \mathrm{fm}^{-3}$. Besides that, the dense enough quark matter could be created in accelerators by heavy ion collisions. Therefore, the study of such a system is not of pure academic interest.

At high density, it is believed that the quarks form a Fermi surface in a very similar way as the electrons do it in metals. Following this similarity further, it is natural to ask whether there is an analogue of the superconductivity in the cold quark-gluon plasma. As in metals, the presence of the Fermi surface should considerably increase the density of states of low-energy quasiparticles. As a result, an arbitrarily small attractive interaction in the diquark channel would be sufficient to create the Cooper pairs and, thus, to produce the (color) superconductivity. In Ref. [6], it was shown that an attractive interaction in the color-antitriplet diquark channel indeed appears. As a result, a non-trivial superconducting order parameter develops and the color gauge symmetry $S U(3)_{c}$ gets broken (by the Higgs mechanism) down to its $S U(2)$ subgroup (in the case of two light quark flavors).

Recently, interest in the study of the color superconducting phase considerably increased [7, 9, 12, 22]. The renewed interest was triggered by the observation that the necessary attractive interaction could be exclusively due to the instanton effects [7 9]. In addition, some other fascinating features of the broken phase, such as color-flavor locking and a new type of chiral symmetry breaking [12], were revealed. From a technical point of view, it was also demonstrated that the renormalization group method is an alternative and a very efficient tool in studying the general properties of dense QCD 1821.

\footnotetext{
*Permanent address.

${ }^{\dagger}$ On leave of absence from Bogolyubov Institute for Theoretical Physics, 252143, Kiev, Ukraine.
} 
While it was believed that the color superconductivity was quite well understood at least qualitatively, the recent results of Refs. 19.20 seem to indicate that there is an inconsistency in all the previous approaches, based on the straightforward use of the simplest Nambu-Jona-Lasinio model and the Bardeen-Cooper-Schrieffer (BCS) type of analysis. The key observation in Refs. [19.20] is that the long range interaction, mediated by the unscreened gluon modes of the magnetic type, may considerably enhance the value of the superconducting order parameter. Such an enhancement would be of great importance in studies of neutron star properties. Note that a somewhat similar conclusion about enhancement was also reached in Ref. [21] where the effective low-energy action of the quark quasiparticles around the Fermi surface was derived.

Being motivated by that observation, in this paper, we reanalyze the problem by using the conventional SchwingerDyson (SD) approach. More precisely, we study the SD equations in dense QCD in the so-called improved rainbow approximation in which the full vertices coincide with the bare ones. Such an approximation includes the one-loop polarization effects in the gluon propagator and, thus, is the simplest non-trivial approximation that takes into account the screening effects. Our expression for the fermion gap derived in the SD approach turns out to be essentially the same as in Ref. [20] where the renormalization group method was mainly used.

This paper is organized as follows. In Sec. II, we present the general structure of the quark propagator that accommodates the color superconductivity. In Sec. III, we discuss the structure of the gluon propagator with the one-loop polarization effects taken into account. The role of the Meissner effect in the color superconducting phase of QCD is discussed in Sec. IV. Then, in Sec. V, we derive the SD equation (in the improved rainbow approximation) for the quark propagator and present an analytical estimate for the solution. The discussion of the results is given in the concluding Section VI. In Appendix A, some formulae used in the derivation of the gap equation are considered. And, in Appendix B, we present an approximate analytical solution to the SD equation.

\section{THE MODEL AND NOTATION}

Here we consider QCD with two light quark flavors $(u$ and $d)$ in the fundamental representation of the $S U(3)_{c}$ color gauge group. At sufficiently large values of the chemical potential, the current masses of the quarks can be neglected[. As a consequence, the model is invariant under the (global) chiral $S U(2)_{L} \times S U(2)_{R}$ transformations.

Following the findings of Ref. [6], we are interested in studying the diquark condensates $\varepsilon^{i j} \varepsilon_{a b 3}\left\langle\left(\psi_{a}^{i}\right)^{T} C \psi_{b}^{j}\right\rangle$ and $\varepsilon^{i j} \varepsilon_{a b 3}\left\langle\left(\psi_{a}^{i}\right)^{T} C \gamma_{5} \psi_{b}^{j}\right\rangle$, responsible for the color superconductivity (here $a, b$ and $i, j$ are the color and flavor indices, respectively). We note, that in perturbation theory the two condensates are related by $U_{A}(1)$ symmetry. Nonperturbatively, however, the instanton effects are expected to break the latter symmetry [7] 8 ].

In the presence of a large chemical potential, it is commonly assumed that the quarks near the Fermi surface (or rather low-energy quasiparticles) are weakly coupled due to asymptotic freedom [23]. Then, it is natural to analyze the dynamics of diquark pairing by using the SD equations with a perturbative kernel[ [21]. At this point, it is appropriate to mention that the realistic value of the chemical potential for the quark matter in the interior of a neutron star is expected to be somewhere in between 200 and $700 \mathrm{MeV}$ (so that the baryon number density is between about $n_{0}$ and $10 n_{0}$ ). Then, the corresponding value of the coupling constant $\alpha_{s}$ should be between about 0.4 and 0.8 . At these values of $\alpha_{s}$, our calculations, based on the perturbative kernel, may just start to fail. At the same time, since the values of the coupling constant are not too large, it is plausible that the orders of the magnitudes of the main results could still be trusted. We will return to the discussion of this point in more detail in Sec. VI.

Here we should mention that the SD equation approach to the study of color superconductivity in dense QCD was also used in Refs. 6, 17,21,24. In this paper, however, we pay special attention to the effect of the unscreened magnetic modes of gluons.

Instead of working with the standard four component Dirac spinors, in our analysis below, it is convenient to introduce the following eight component Majorana spinors:

$$
\Psi=\frac{1}{\sqrt{2}}\left(\begin{array}{c}
\psi \\
\psi^{C}
\end{array}\right), \quad \psi^{C}=C \bar{\psi}^{T}
$$

\footnotetext{
${ }^{1}$ As is argued in Ref. [12], the strange quark is also sufficiently light to be included in the model. However, we are not interested here in the specific effects of the third flavor. Our prime goal here is to clarify the role of the long range gluon interaction and, thus, it is sufficient for our purposes to deal with the simplest model of two light flavors.

${ }^{2}$ Note that according to Ref. [18, the effect of instantons could be negligible compared to the one-gluon exchange at sufficiently large chemical potential.
} 
where $C$ is a charge conjugation matrix, defined by $C^{-1} \gamma_{\mu} C=-\gamma_{\mu}^{T}$ and $C=-C^{T}$. In the new notation, the inverse fermion propagator (defined over the true vacuum) that accommodates the possibility of the diquark condensation reads

$$
(G(p))^{-1}=-i\left(\begin{array}{cc}
A(p)\left[\left(p_{0}+\mu\right) \gamma^{0}+B(p) \not \mathfrak{p}\right] & \Delta \\
\widetilde{\Delta} & A(p)\left[\left(p_{0}-\mu\right) \gamma^{0}+B(p) \not \mathfrak{p}\right]
\end{array}\right)
$$

where $\tilde{\Delta}=\gamma^{0} \Delta^{\dagger} \gamma^{0}$ and $\Delta_{a b}^{i j} \equiv \varepsilon^{i j} \varepsilon_{a b 3} \Delta_{3}$ (note that $\Delta_{3}$ is a matrix in the Dirac space). By definition, $\ddot{p}=-\vec{p} \cdot \vec{\gamma}$.

Now, after inverting the expression in Eq. (21), we arrive at the following propagator:

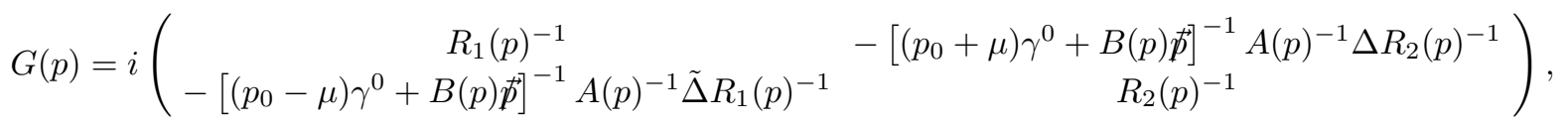

where

$$
\begin{aligned}
& R_{1}(p)=A(p)\left[\left(p_{0}+\mu\right) \gamma^{0}+B(p) \hat{p}\right]-\Delta\left[\left(p_{0}-\mu\right) \gamma^{0}+B(p) \hat{p}\right]^{-1} A(p)^{-1} \tilde{\Delta} \\
& R_{2}(p)=A(p)\left[\left(p_{0}-\mu\right) \gamma^{0}+B(p) \hat{p}\right]-\tilde{\Delta}\left[\left(p_{0}+\mu\right) \gamma^{0}+B(p) \hat{p}\right]^{-1} A(p)^{-1} \Delta .
\end{aligned}
$$

In Sec. V, we use the expression for the propagator in Eq. (3) as an anzatz for the solution to the SD equation. Notice that, with the given choice of the condensate, the most general structure of the wave function renormalizations $A(p)$ and $B(p)$ (in flavor and color spaces) has to be as follows: $A_{a b}^{i j}(p)=a(p)\left(\delta_{a b}-\delta_{a 3} \delta_{b 3}\right) \delta^{i j}+\bar{a}(p) \delta_{a 3} \delta_{b 3} \delta^{i j}$ and $B_{a b}^{i j}(p)=b(p)\left(\delta_{a b}-\delta_{a 3} \delta_{b 3}\right) \delta^{i j}+\bar{b}(p) \delta_{a 3} \delta_{b 3} \delta^{i j}$, where $a(p), \bar{a}(p)$ and $b(p), \bar{b}(p)$ are some scalar functions.

\section{VACUUM POLARIZATION IN THE GLUON PROPAGATOR}

In this section we discuss the vacuum polarization effects in the gluon propagator.

The calculation of the polarization tensor was performed by others [25] 27] and we are not going to repeat it here. Instead we present the final result and comment on its essential features.

Let us start from the expression for the inverse gluon propagator in a covariant gauge with polarization effects taken into account. Its explicit form is given by

$$
\left(\mathcal{D}_{\mu \nu}^{A B}\left(k_{0}, \vec{k}\right)\right)^{-1}=i \delta^{A B} k^{2} P_{\mu \nu}^{\perp}+i \delta^{A B} \frac{k^{2}}{d} P_{\mu \nu}^{\|}+i \delta^{A B} \Pi_{\mu \nu}\left(k_{0}, \vec{k}\right) .
$$

where the color indices $A, B=1,2, \ldots, 8$, and the standard projection operators $P_{\mu \nu}^{\perp}$ and $P_{\mu \nu}^{\|}$,

$$
P_{\mu \nu}^{\perp}=g_{\mu \nu}-\frac{k_{\mu} k_{\nu}}{k^{2}}, \quad \text { and } \quad P_{\mu \nu}^{\|}=\frac{k_{\mu} k_{\nu}}{k^{2}},
$$

were introduced. The gauge fixing parameter $d$ in Eq. (5) is arbitrary.

At a finite density of quarks, the Lorentz symmetry is explicitly broken and, as a result, the polarization tensor $\Pi_{\mu \nu}$ could also contain the third tensor structure [28]:

$$
P_{\mu \nu}^{u}=\frac{k_{\mu} k_{\nu}}{k^{2}}-\frac{k_{\mu} u_{\nu}+u_{\mu} k_{\nu}}{(u \cdot k)}+\frac{u_{\mu} u_{\nu}}{(u \cdot k)^{2}} k^{2},
$$

where $u_{\mu}=(1,0,0,0)$. This last tensor, similarly to $P_{\mu \nu}^{\perp}$, is transverse, $k^{\mu} P_{\mu \nu}^{u}=0$. The set of all the three tensors, introduced so far, satisfy the following set of multiplication rules:

$$
\begin{array}{rlrl}
P^{\perp} P^{\perp}=P^{\perp}, & P^{\|} P^{\|}=P^{\|}, & P^{u} P^{u}=\left(\frac{k^{2}}{(u \cdot k)^{2}}-1\right) P^{u}, \\
P^{\perp} P^{\|}=P^{\|} P^{\perp}=0, & P^{\|} P^{u}=P^{u} P^{\|}=0, & P^{u} P^{\perp} & =P^{\perp} P^{u}=P^{u},
\end{array}
$$

where we assume that the operator products are defined by the appropriate contractions involving the Minkowski metric. While working with the gluon propagator, it is much more convenient to use the following set of mutually orthogonal projection operators: 


$$
\begin{array}{cl}
O^{(1)}=P^{\perp}+\frac{(u \cdot k)^{2}}{(u \cdot k)^{2}-k^{2}} P^{u}, & O^{(2)}=-\frac{(u \cdot k)^{2}}{(u \cdot k)^{2}-k^{2}} P^{u}, \quad O^{(3)}=P^{\|}, \\
O^{(1)} O^{(1)}=O^{(1)}, & O^{(2)} O^{(2)}=O^{(2)}, \quad O^{(3)} O^{(3)}=O^{(3)}, \\
O_{\mu \nu}^{(1)}+O_{\mu \nu}^{(2)}+O_{\mu \nu}^{(3)}=g_{\mu \nu}, & O^{(i)} O^{(j)}=0, \quad \text { for } i \neq j .
\end{array}
$$

At finite chemical potential, in the one-loop approximation, the calculation of the polarization tensor reduces to evaluating the contribution of three diagrams: with gluons, quarks and ghosts running in the loop [25], respectively. In the hard dense loop approximation, the result for the polarization tensor is given by [25] 27],

$$
\begin{aligned}
& \Pi^{00}\left(k_{0}, \vec{k}\right)=\Pi_{l}\left(k_{0}, \vec{k}\right), \\
& \Pi^{0 i}\left(k_{0}, \vec{k}\right)=k_{0} \frac{k^{i}}{|\vec{k}|^{2}} \Pi_{l}\left(k_{0}, \vec{k}\right), \\
& \Pi^{i j}\left(k_{0}, \vec{k}\right)=\left(\delta^{i j}-\frac{k^{i} k^{j}}{|\vec{k}|^{2}}\right) \Pi_{t}\left(k_{0}, \vec{k}\right)+\frac{k^{i} k^{j}}{|\vec{k}|^{2}} \frac{k_{0}^{2}}{|\vec{k}|^{2}} \Pi_{l}\left(k_{0}, \vec{k}\right),
\end{aligned}
$$

where

$$
\begin{aligned}
& \Pi_{l}\left(k_{0}, \vec{k}\right)=2 M^{2}\left(\frac{k_{0}}{2|\vec{k}|} \ln \left|\frac{k_{0}+|\vec{k}|}{k_{0}-|\vec{k}|}\right|-1-i \pi \frac{k_{0}}{2|\vec{k}|} \theta\left(-k^{2}\right)\right), \\
& \Pi_{t}\left(k_{0}, \vec{k}\right)=M^{2}-\frac{k^{2}}{2|\vec{k}|^{2}} \Pi_{l}\left(k_{0}, \vec{k}\right) .
\end{aligned}
$$

Here we use the notation $M^{2}=\left(g_{s} \mu \sqrt{N_{f}} / 2 \pi\right)^{2}$. What is remarkable about this result is that it coincides with the polarization tensor derived in the framework of classical transport theory of dense Yang-Mills plasma [26.27.

Notice that the polarization tensor in Eq. (10) has a nonzero imaginary part for space-like gluon momenta. This imaginary part is responsible for the so-called Landau damping of the gluon field with space-like momenta. Also, it is responsible for the quark damping around the Fermi surface [25,29]. In our analysis below, however, we neglect the effects of quark damping. Such an approximation is partly justified by the fact that the damping rate goes to zero linearly as one approaches the Fermi surface 29.

The gluon polarization tensor in Eq. (10) is transverse

$$
k^{\mu} \Pi_{\mu \nu}\left(k_{0}, \vec{k}\right)=0 .
$$

Because of this last property, it is natural to try to express the real part of the polarization tensor in terms of the two transverse projection operators, $O^{(1)}$ and $O^{(2)}$, introduced earlier. This turns out to be an easy task and the result takes the following nice form:

$$
\Pi_{\mu \nu}=-O_{\mu \nu}^{(1)} \Pi_{t}+2 O_{\mu \nu}^{(2)}\left(\Pi_{t}-M^{2}\right) .
$$

With this expression at hand, we rewrite the inverse gluon propagator in Eq. (5) as

$$
\left(\mathcal{D}_{\mu \nu}^{A B}\left(k_{0}, \vec{k}\right)\right)^{-1}=i \delta^{A B}\left(k^{2}-\Pi_{t}\right) O_{\mu \nu}^{(1)}+i \delta^{A B}\left(k^{2}+2 \Pi_{t}-2 M^{2}\right) O_{\mu \nu}^{(2)}+i \delta^{A B} \frac{k^{2}}{d} O_{\mu \nu}^{(3)} .
$$

Then, by making use of the properties of the projection operators, we could easily invert this expression and arrive at the final form of the gluon propagator that includes the (one-loop) screening effects

$$
\mathcal{D}_{\mu \nu}^{A B}\left(k_{0}, \vec{k}\right)=-i \delta^{A B} \frac{1}{k^{2}-\Pi_{t}} O_{\mu \nu}^{(1)}-i \delta^{A B} \frac{1}{k^{2}+2 \Pi_{t}-2 M^{2}} O_{\mu \nu}^{(2)}-i \delta^{A B} \frac{d}{k^{2}} O_{\mu \nu}^{(3)} .
$$

This representation of the gluon propagator, in addition to its convenience for calculations, also has another advantage. It allows to separate different gluon modes in a very simple way. Indeed, to make such a separation, we just need to project the general gluon state by using one of the three operators, $O^{(1)}, O^{(2)}$ or $O^{(3)}$, respectively. As a result we come to the following types of modes: $a_{\mu}^{(m)}=O_{\mu \nu}^{(1)} A^{\nu}$ (magnetic), $a_{\mu}^{(e)}=O_{\mu \nu}^{(2)} A^{\nu}$ (electric) and $a_{\mu}^{(\|)}=O_{\mu \nu}^{(3)} A^{\nu}$ (longitudinal). The name "magnetic" here reflects the point that the corresponding projection operator does not have 
the electric components $O_{\nu 0}^{(1)}=O_{0 \nu}^{(1)}=0$. Apparently, the rest of the transverse modes are the "electric" modes. As for the unphysical longitudinal mode, its definition is standard.

In the rest of this Section, we will consider the part of the gluon propagator connected with the unbroken, $S U(2)$, subgroup of the $S U(3)_{c}$, i.e., $A, B=1,2,3$. The part of the gluon propagator connected with the broken generators, $A, B=4, \ldots, 8$, will be considered in the next Section.

Because of a specific dynamics of diquark pairing in the vicinity of the Fermi surface, it is believed that the most relevant gluons mediating the interaction are those with the space like momenta. Indeed, when the quarks around the Fermi surface scatter, their energy does not change much, while their momentum could change for as much as $2 p_{F}$. Thus, we could mimic the screening effects by the following asymptotic form of $\Pi_{t}$ in the region $|\vec{k}| \gg k_{0}$ :

$$
\Pi_{t}\left(k_{0}, \vec{k}\right) \simeq 2 M^{2} \frac{k_{0}^{2}}{|\vec{k}|^{2}}\left(1-\frac{1}{3} \frac{k_{0}^{2}}{|\vec{k}|^{2}}+\ldots\right)+i \pi M^{2} \frac{k_{0}}{2|\vec{k}|}\left(1-\frac{k_{0}^{2}}{|\vec{k}|^{2}}\right) \theta\left(|\vec{k}|^{2}-k_{0}^{2}\right) .
$$

In fact, this is a very good approximation even for the values of the ratio $k_{0} /|\vec{k}|$ as large as 0.8 at which the deviation of the asymptote from the exact expression is of order of $5 \%$. If we keep only the leading term in Eq. (17), the same magnitude of deviation is reached at about $k_{0} /|\vec{k}| \simeq 0.4$.

By substituting this asymptote into the gluon propagator, we see that the magnetic mode $a_{\mu}^{(m)}$ produces the long-range interaction, while the electric mode $a_{\mu}^{(e)}$ gets screened out. In what follows, it is convenient to use the representation of the gluon propagator in terms of spectral densities:

$$
i \mathcal{D}_{\mu \nu}^{A B}\left(k_{0}, \vec{k}\right) \simeq \delta^{A B} \lim _{\varepsilon \rightarrow 0} \frac{1}{2 \pi} \int_{-\infty}^{\infty} \frac{d z}{k_{0}-z+i \varepsilon}\left[\rho_{m}(z, \vec{k}) O_{\mu \nu}^{(1)}+\rho_{e}(z, \vec{k}) O_{\mu \nu}^{(2)}+\rho_{\|}(z, \vec{k}) O_{\mu \nu}^{(3)}\right],
$$

where

$$
\begin{aligned}
\rho_{m}(z, \vec{k}) & \simeq \frac{\pi M^{2} z|\vec{k}|}{|\vec{k}|^{6}+(\pi / 2)^{2} M^{4} z^{2}} \theta\left(|\vec{k}|^{2}-z^{2}\right), \\
\rho_{e}(z, \vec{k}) & \simeq 2 \pi \operatorname{sgn}(z) \delta\left(z^{2}-|\vec{k}|^{2}-2 M^{2}\right), \\
\rho_{\|}(z, \vec{k}) & =2 \pi d \operatorname{sgn}(z) \delta\left(z^{2}-|\vec{k}|^{2}\right) .
\end{aligned}
$$

Therefore, by making use of the representation in Eq. (18), we arrive at the following expression for the gluon propagator in Euclidean space $\left(k_{0}=i k_{4}\right)$ :

$$
i \mathcal{D}_{\mu \nu}^{A B}\left(i k_{4}, \vec{k}\right) \simeq-\delta^{A B} \frac{|\vec{k}|}{|\vec{k}|^{3}+\pi M^{2}\left|k_{4}\right| / 2} O_{\mu \nu}^{(1)}-\delta^{A B} \frac{1}{k_{4}^{2}+|\vec{k}|^{2}+2 M^{2}} O_{\mu \nu}^{(2)}-\delta^{A B} \frac{d}{k_{4}^{2}+|\vec{k}|^{2}} O_{\mu \nu}^{(3)},
$$

where $A, B=1,2,3$. This propagator correctly describes the gluons in the one-loop approximation in the soft momentum region. In Sec. $\mathrm{V}$, we shall use it in the SD equation for the quark propagator. As we shall see there, because of the long range interaction mediated by the first term in the propagator in Eq. (22), the result for the order parameter is going to be quite different from that obtained in the theories with local interactions [12, 18]. Notice, that according to the arguments of Refs. [19.20], the magnetic modes of the unbroken $S U(2)$ subgroup of $S U(3)_{c}$ should develop no screening even after taking into account nonperturbative effects.

\section{THE MEISSNER EFFECT}

In this section, we study the role of the Meissner effect in color superconducting phase of dense QCD. Based on pure symmetry arguments, it is clear that when the color $S U(3)$ symmetry spontaneously breaks down to $S U(2)$, five out of eight gluons should get masses by the Higgs mechanism.

To estimate the value of the Higgs-like gluon mass, we need to calculate the appropriate contribution to the vacuum polarization tensor that results from the non-diagonal term in the fermion propagator in Eq. (3). To get a rough estimate of the mass, we could completely neglect the effects of the wave function renormalizations (in the next Section, we will give a justification for that). Then, we get the following order of magnitude expression:

$$
\mathcal{P}(0)=M_{0}^{2} \simeq \frac{\alpha_{s}}{\pi^{2}} \int \frac{d^{3} \vec{q} d q_{4} \mu^{2}\left|\Delta^{(-)}\right|^{2}}{\left[(\mu-|\vec{q}|)^{2}+q_{4}^{2}+\left|\Delta^{(-)}\right|^{2}\right]^{2}} \simeq \frac{\alpha_{s}}{\pi} \mu^{2},
$$


where the order parameter $\Delta^{(-)}$is defined in Sec. $\mathrm{V}$ [see Eq. (42)]. This seems to suggest that the five gluons obtain relatively large masses (of order $\alpha_{s} \mu^{2}$ ) due to the Meissner effect. As we show in a moment, however, this conclusion is not quite right.

Before going into more details, it is instructive to remind a few facts about the Meissner effect of the low-temperature superconductivity in ordinary (non-relativistic) metals [30]. As is well known, there exist two characteristic scales in the theory: the coherence length, $\xi$, and the London magnetic penetration depth, $\lambda_{L}$. For our purposes here, it is sufficient to recall that while the coherence length, as $T \rightarrow 0$, is directly related to the value of the superconducting order parameter $(\xi \sim 1 /|\Delta|)$, the London penetration depth is independent of $\Delta$ and is given in terms of the mass and the density of the electrons $\left(\lambda_{L} \sim \sqrt{m / n_{e l}}\right)$.

The London penetration depth $\lambda_{L}$ coincides with the actual magnetic penetration depth $\lambda$ only in type II superconductors with $\lambda_{L} \gg \xi: 1 / \lambda_{L}$ coincides with the value of the running Higgs-like mass of a plasmon at zero momentum and the region of small momenta yields the dominant contribution to the actual penetration depth in type II superconductors. On the other hand, in type I superconductors, it is the running Higgs-like mass at momenta $\Delta \ll k \ll \mu$ that yields the dominant contribution to $\lambda$. In this case $\lambda=\lambda_{P}$ where $\lambda_{P}$ is the Pippard penetration $\operatorname{depth}$ ]: $\lambda_{P} \sim\left(\lambda_{L}^{2} \xi\right)^{1 / 3}$ 30.31.

Regarding the cold dense quark matter, it can hardly be a type II superconductor because, as we shall see from our final estimate for the gap in Eq. (42), $\left|\Delta^{(-)}\right|$is $\left|\Delta^{(-)}\right| \ll M_{0}$, implying that $\lambda_{L} \ll \xi$. Therefore, most likely, it is a type I superconductor. This means that the actual penetration depth of a magnetic field is given not by the London expression but by the Pippard one. Technically the difference comes from the fact that the zero-momentum expression in Eq. (23), related to the definition of the London penetration depth, is valid only in a very small region of momenta, $|\vec{k}| \ll\left|\Delta^{(-)}\right|$. In the most important intermediate region, $\left|\Delta^{(-)}\right| \ll|\vec{k}| \ll \mu$, however, the expression has the following asymptotic behavior [31]:

$$
\mathcal{P}(k) \simeq M_{0}^{2} \frac{\left|\Delta^{(-)}\right|}{|\vec{k}|}
$$

Since the relevant momenta for the Meissner effect are of order $\lambda^{-1} \gg\left|\Delta^{(-)}\right|$, from Eq. (24), we indeed see that the penetration depth is equal to $\lambda_{P} \sim\left(M_{0}^{2}\left|\Delta^{(-)}\right|\right)^{-1 / 3}$ rather than $\lambda_{L} \sim 1 / M_{0}$.

Now, what the role does the Meissner effect play in the perturbative kernel of the SD equation? To answer this question, let us consider how the corresponding propagators of magnetic gluons are going to be modified by the Meissner effect. Apparently, we could still use the representation for the propagators as in Eq. (18). The spectral density, though, should be modified appropriately:

$$
\rho_{m}^{(M)}(z, \vec{k}) \simeq \frac{\pi M^{2} z|\vec{k}|}{\left(|\vec{k}|^{3}+M_{0}^{2}\left|\Delta^{(-)}\right|\right)^{2}+(\pi / 2)^{2} M^{4} z^{2}} \theta\left(|\vec{k}|^{2}-z^{2}\right),
$$

where the superscript $M$ stays here for the "Meissner effect". After simple calculations, we arrive at the following expression for the corresponding propagator in Euclidean space $\left(k_{0}=i k_{4}\right)$ :

$$
i \mathcal{D}_{\mu \nu}^{(M) A B}\left(i k_{4}, \vec{k}\right) \simeq-\delta^{A B} \frac{|\vec{k}|}{|\vec{k}|^{3}+M_{0}^{2}\left|\Delta^{(-)}\right|+\pi M^{2}\left|k_{4}\right| / 2} O_{\mu \nu}^{(1)}+\ldots, \quad \text { for } \quad A, B=4, \ldots, 8,
$$

where the ellipsis denote the same electric and longitudinal contributions as in Eq. (22). By comparing the propagators of magnetic modes in Eqs. (22) and (26), we see that the Meissner effect could be accounted by the formal replacement $k_{4} \rightarrow k_{4}+c\left|\Delta^{(-)}\right|$with $c=O(1)$ in the magnetic term of the five propagators in Eq. (26). Therefore, it is obvious that the difference between the propagators in Eqs. (22) and (26) could become important only in the range of momenta $\left|k_{4}\right| \lesssim\left|\Delta^{(-)}\right|$and $|\vec{k}| \lesssim\left(M_{0}^{2}\left|\Delta^{(-)}\right|\right)^{1 / 3}$. In the next section, we shall see that the mentioned region of gluon momenta is not large enough to modify the leading asymptote of the solution to the gap equation.

\footnotetext{
${ }^{3}$ We would like to thank S. Hsu for pointing our attention to the difference between the definitions of the London and the Pippard penetration depths.
} 


\section{SCHWINGER-DYSON EQUATION IN DENSE QCD}

In this section we derive the SD equation in dense QCD, ignoring the masses of quarks. All the necessary constituents of such an equation were given in the previous three sections. In the improved rainbow approximation, the equation reads

$$
\begin{aligned}
\left(G_{a b}(p)\right)^{-1}= & \left(G_{a b}^{(0)}(p)\right)^{-1}+4 \pi \alpha_{s} \int \frac{d^{4} q}{(2 \pi)^{4}}\left(\begin{array}{cc}
\gamma^{\mu} & 0 \\
0 & -\gamma^{\mu}
\end{array}\right) \sum_{a^{\prime}, b^{\prime}} \sum_{A=1}^{3} T_{a^{\prime} a}^{A} G_{a^{\prime} b^{\prime}}(q) T_{b^{\prime} b}^{A}\left(\begin{array}{cc}
\gamma^{\nu} & 0 \\
0 & -\gamma^{\nu}
\end{array}\right) \mathcal{D}_{\mu \nu}(q-p) \\
& +4 \pi \alpha_{s} \int \frac{d^{4} q}{(2 \pi)^{4}}\left(\begin{array}{cc}
\gamma^{\mu} & 0 \\
0 & -\gamma^{\mu}
\end{array}\right) \sum_{a^{\prime}, b^{\prime}} \sum_{A=4}^{8} T_{a^{\prime} a}^{A} G_{a^{\prime} b^{\prime}}(q) T_{b^{\prime} b}^{A}\left(\begin{array}{cc}
\gamma^{\nu} & 0 \\
0 & -\gamma^{\nu}
\end{array}\right) \mathcal{D}_{\mu \nu}^{(M)}(q-p),
\end{aligned}
$$

where $\mathcal{D}_{\mu \nu}(k)$ is the propagator of gluons that correspond to the unbroken $S U(2)$ subgroup [see Eq. (22)], while $\mathcal{D}_{\mu \nu}^{(M)}(k)$ is the propagator of those five gluons whose magnetic modes are modified by the Meissner effect [see Eq. (26)]. Notice, that the overall factor $\delta^{A B}$ is omitted in the definition of the propagators here. Regarding the rest of notation, $G_{a b}(p)$ is the full fermion propagator, and $G_{a b}^{(0)}(p)$ is the perturbative one. With our choice of the order parameter orientation in the color space, $\Delta_{a b} \sim \varepsilon_{a b 3}$, the explicit form of the generators of the unbroken color subgroup reads

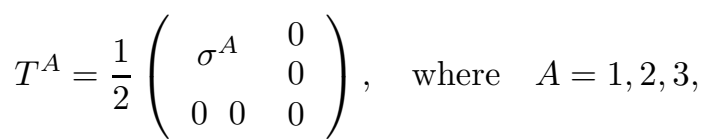

and $\sigma^{A}$ are the Pauli matrices. Then, by making use of the well known identity for the Pauli matrices, we arrive at the following result for the summation over $A$ :

$$
\sum_{A=1}^{3} T_{a^{\prime} a}^{A} T_{b^{\prime} b}^{A}=\frac{1}{2}\left(\delta_{a^{\prime} b}-\delta_{a^{\prime} 3} \delta_{b 3}\right)\left(\delta_{a b^{\prime}}-\delta_{a 3} \delta_{b^{\prime} 3}\right)-\frac{1}{4}\left(\delta_{a a^{\prime}}-\delta_{a 3} \delta_{a^{\prime} 3}\right)\left(\delta_{b b^{\prime}}-\delta_{b 3} \delta_{b^{\prime} 3}\right) .
$$

By making use of the identity for all eight generators of $S U(3)$ :

$$
\sum_{A=1}^{8} T_{a^{\prime} a}^{A} T_{b^{\prime} b}^{A}=\frac{1}{2} \delta_{a^{\prime} b} \delta_{a b^{\prime}}-\frac{1}{6} \delta_{a a^{\prime}} \delta_{b b^{\prime}}
$$

we also obtain the following one:

$$
\sum_{A=4}^{8} T_{a^{\prime} a}^{A} T_{b^{\prime} b}^{A}=\frac{1}{12} \delta_{a a^{\prime}} \delta_{b b^{\prime}}+\frac{1}{2}\left(\delta_{a^{\prime} b} \delta_{a 3} \delta_{b^{\prime} 3}+\delta_{a b^{\prime}} \delta_{a^{\prime} 3} \delta_{b 3}\right)-\frac{1}{4}\left(\delta_{a a^{\prime}} \delta_{b 3} \delta_{b^{\prime} 3}+\delta_{b b^{\prime}} \delta_{a 3} \delta_{a^{\prime} 3}\right)-\frac{1}{4} \delta_{a 3} \delta_{b 3} \delta_{a^{\prime} 3} \delta_{b^{\prime} 3}
$$

Now, upon inserting the identities (29) and (31) along with the expression for the fermion propagator (3) into Eq. (27), we arrive at the set of integral equations:

$$
\begin{aligned}
a(p) \Delta^{( \pm)}(p) & =\frac{\pi \alpha_{s}}{6} \int \frac{d^{4} q}{(2 \pi)^{4}} \frac{1}{a(q)}\left(9 \mathcal{D}_{\mu \nu}(q-p)-\mathcal{D}_{\mu \nu}^{(M)}(q-p)\right) \\
& \times\left[\frac{\Delta^{(-)}(q) \operatorname{Tr}\left(\gamma^{\mu} \Lambda_{q}^{(+)} \gamma^{\nu} \Lambda_{p}^{( \pm)}\right)}{q_{0}^{2}-(b|\vec{q}|-\mu)^{2}-\left|\Delta^{(-)}\right|^{2}}+\frac{\Delta^{(+)}(q) \operatorname{Tr}\left(\gamma^{\mu} \Lambda_{q}^{(-)} \gamma^{\nu} \Lambda_{p}^{( \pm)}\right)}{q_{0}^{2}-(b|\vec{q}|+\mu)^{2}-\left|\Delta^{(+)}\right|^{2}}\right], \\
(a-1)\left(p_{0}+\mu\right) & \mp(a b-1)|\vec{p}|=-\frac{\pi \alpha_{s}}{6} \int \frac{d^{4} q}{(2 \pi)^{4}} \frac{1}{a(q)}\left(3 \mathcal{D}_{\mu \nu}(q-p)+\mathcal{D}_{\mu \nu}^{(M)}(q-p)\right) \\
& \times\left[\frac{\left(q_{0}+b|\vec{q}|-\mu\right) \operatorname{Tr}\left(\gamma^{0} \gamma^{\mu} \Lambda_{q}^{(+)} \gamma^{0} \gamma^{\nu} \Lambda_{p}^{( \pm)}\right)}{q_{0}^{2}-(b|\vec{q}|-\mu)^{2}-\left|\Delta^{(-)}\right|^{2}}+\frac{\left(q_{0}-b|\vec{q}|-\mu\right) \operatorname{Tr}\left(\gamma^{0} \gamma^{\mu} \Lambda_{q}^{(-)} \gamma^{0} \gamma^{\nu} \Lambda_{p}^{( \pm)}\right)}{q_{0}^{2}-(b|\vec{q}|+\mu)^{2}-\left|\Delta^{(+)}\right|^{2}}\right] \\
(\bar{a}-1)\left(p_{0}+\mu\right) & \mp(\bar{a} \bar{b}-1)|\vec{p}|=-\frac{2 \pi \alpha_{s}}{3} \int \frac{d^{4} q}{(2 \pi)^{4}} \frac{1}{\bar{a}(q)} \mathcal{D}_{\mu \nu}^{(M)}(q-p) \\
\times & {\left[\frac{\operatorname{Tr}\left(\gamma^{0} \gamma^{\mu} \Lambda_{q}^{(+)} \gamma^{0} \gamma^{\nu} \Lambda_{p}^{( \pm)}\right)}{q_{0}-\bar{b}|\vec{q}|+\mu}+\frac{\operatorname{Tr}\left(\gamma^{0} \gamma^{\mu} \Lambda_{q}^{(-)} \gamma^{0} \gamma^{\nu} \Lambda_{p}^{( \pm)}\right)}{q_{0}+\bar{b}|\vec{q}|+\mu}\right] }
\end{aligned}
$$


Here, Tr denotes the trace in Dirac indices, and $\Delta^{( \pm)}$are complex functions of momentum, defined by eitherf $\Delta_{3}(p)=$ $a(p)\left[\Delta^{(+)}(p) \Lambda_{p}^{(+)}+\Delta^{(-)}(p) \Lambda_{p}^{(-)}\right]$(parity odd order parameter) or $\Delta_{3}(p)=a(p) \gamma_{5}\left[\Delta^{(+)}(p) \Lambda_{p}^{(+)}+\Delta^{(-)}(p) \Lambda_{p}^{(-)}\right]$ (parity even order parameter), while

$$
\Lambda_{p}^{(\mp)} \equiv \frac{1}{2}\left(1 \mp \frac{\vec{\alpha} \cdot \vec{p}}{|\vec{p}|}\right)
$$

are the free quark (antiquark) on-shell projectors [19,32,33, 3 .

Let us start our analysis by considering the equations for the wave function renormalizations (33) and (34) first. In order to get a rough estimate for the wave function renormalizations, it is sufficient to substitute $a=b=\bar{a}=\bar{b}=1$ along with $\Delta^{( \pm)}=0$ into the right hand side of Eqs. (33) and (34). Then, it is straightforward to show that (i) the wave function renormalization of the temporal and the spatial parts of the fermion kinetic terms are not equal, and (ii) the one-loop corrections to $a(p)$ and $b(p)$ develop logarithmic divergences at $p_{4}=0$ and $|\vec{p}|=\mu$. As is clear, these logarithmic divergences at the Fermi surface are going to be removed when a non-zero order parameter $\Delta^{(-)}$is reintroduced. Notice that, because of the Meissner effect, no infrared divergences develop in $\bar{a}(p)$ and $\bar{b}(p)$ either. At the end, we obtain the following estimates:

$$
a, b, \bar{a}, \bar{b} \simeq 1+\text { Const } \cdot \alpha_{s}(\mu) \ln \frac{\mu}{\left|\Delta^{(-)}\right|} .
$$

By taking into account the expected value of the order parameter [see Eq. (42) below], we actually see that all wave function renormalizations are close to 1 if the coupling constant $\alpha_{s}(\mu)$ is weak. Therefore, we conclude that, in the leading order approximation, it is justified to neglect the wave function renormalization effects.

The fact that $a \approx b \approx \bar{a} \approx \bar{b} \approx 1$ considerably simplifies the study of the gap equation (32). However, one still has a rather complicated set of two coupled integral equations for $\Delta^{(-)}$and $\Delta^{(+)}$, respectively. At this point, it is important to notice that only $\Delta^{(-)}$defines the gap in the quasiparticle spectrum around the Fermi surface. Also, in the leading order approximation, it is sufficient to keep only those terms on the right hand side of Eq. (32) which become singular at the Fermi surface as $\Delta^{(-)} \rightarrow 0$. Then, we see that the equation for $\Delta^{(-)}(p)$ decouples:

$$
\Delta^{(-)}(p)=\frac{\pi \alpha_{s}}{6} \int \frac{d^{4} q}{(2 \pi)^{4}}\left(9 \mathcal{D}_{\mu \nu}(q-p)-\mathcal{D}_{\mu \nu}^{(M)}(q-p)\right) \frac{\Delta^{(-)}(q) \operatorname{Tr}\left(\gamma^{\mu} \Lambda_{q}^{(+)} \gamma^{\nu} \Lambda_{p}^{(-)}\right)}{q_{0}^{2}-(|\vec{q}|-\mu)^{2}-\left|\Delta^{(-)}\right|^{2}} .
$$

We further simplify the gap equation by assuming that $\Delta^{(-)}(p) \equiv \Delta^{(-)}\left(p_{4}, \vec{p}_{F}\right)$ with $\vec{p}_{F}=(0,0, \mu)$. This approximation with the order parameter being a function of only $p_{4}$ is partly justified by the structure of the magnetic (dominant) part of the perturbative kernel in the SD equation. Indeed, in the vicinity of the Fermi surface, the dependence of the kernel is more sensitive to changes in $p_{4}$ than to changes in $|\vec{p}|$. This is due to the fact that while the dependence on $p_{4}$ comes from the linear term in the denominator of the propagator of the magnetic modes in Eqs. (22) and (26), the dependence on $|\vec{p}|$ comes from the cubic term.

In order to linearize Eq. (37), we substitute $\left.\Delta^{(-)}(p) \rightarrow \Delta^{(-)} \equiv \Delta^{(-)}\left(p_{4}\right)\right|_{p_{4}=0}$ in the denominator. Then, after taking the trace over the Dirac indices and switching to the Euclidean space $\left(q_{0}=i q_{4}\right)$, the integral over the angular coordinates in Eq. (37) can be done exactly (see Appendix A for details). After performing the subsequent approximate integration over the absolute value of the spatial momentum, we finally arrive at

$$
\Delta^{(-)}\left(p_{4}\right)=\frac{\alpha_{s}}{72 \pi} \int_{-\infty}^{\infty} d q_{4} \frac{\Delta^{(-)}\left(q_{4}\right)}{\sqrt{q_{4}^{2}+\left|\Delta^{(-)}\right|^{2}}}\left(9 \ln \frac{2(2 \mu)^{3}}{\pi M^{2}\left|q_{4}-p_{4}\right|}-\ln \frac{2(2 \mu)^{3}}{2 M_{0}^{2}\left|\Delta^{(-)}\right|+\pi M^{2}\left|q_{4}-p_{4}\right|}+12 \ln \frac{(2 \mu)^{2}}{2 M^{2}}+12 d\right),
$$

As is easy to trace back, the first two terms in this expression come from the interaction mediated by the magnetic modes: while the first one is connected with the three gluons of the unbroken $S U(2)$ subgroup, the second term comes from the interaction mediated by the five gluons subject to the Meissner effect. The third term in Eq. (38) is connected with the electric modes, and the last term comes from the longitudinal modes. By noticing that the

\footnotetext{
${ }^{4}$ Notice that we factored out the renormalization of the wave function $a(p)$ in the definition of $\Delta_{3}(p)$.

${ }^{5}$ In our notations, $\Delta^{(-)}$is the same as $\left(\Delta_{1}\right)^{*}$ of Ref. 32].
} 
main contribution to the right hand side comes from the region of momenta $\left|\Delta^{(-)}\right| \ll\left|q_{4}\right| \ll \mu$, it is obvious that the Meissner effect is of no importance in this gap equation. Therefore, by putting $M_{0}=0$, we arrive at

$$
\Delta^{(-)}\left(p_{4}\right) \simeq \frac{\alpha_{s}}{9 \pi} \int \frac{d q_{4} \Delta^{(-)}\left(q_{4}\right)}{\sqrt{q_{4}^{2}+\left|\Delta^{(-)}\right|^{2}}} \ln \frac{\Lambda}{\left|q_{4}-p_{4}\right|},
$$

where $\Lambda \equiv e^{3 d / 2}(2 \mu)^{6} /\left(\sqrt{2} \pi M^{5}\right)=e^{3 d / 2}(4 \pi)^{3 / 2} \mu / \alpha^{5 / 2}$.

Notice that, because of the absence of the logarithmic factor in front of the gauge parameter in Eq.(38), the longitudinal gluon modes become relevant only in the next-to-leading order. Here, however, we keep their contribution in order to estimate the size of possible corrections to the magnitude of the order parameter due to subleading effects [see the discussion following Eq. (42)]. The contribution of the electric modes is also subleading. However, because of the large logarithm $\ln \left(\mu^{2} / M^{2}\right) \sim \ln \left(1 / \alpha_{s}\right)$, their contribution to the gap is important at asymptotically high density of quark matter when the coupling $\alpha_{s}(\mu)$ is weak [see the discussion after Eq. (42)].

To get a rough estimate of the order of the gap, we could just put a sharp ultraviolet cut-off at $q_{4}=\mu$ and then, assuming that $\Delta^{(-)}\left(q_{4}\right) \approx \Delta^{(-)}=$Const in this region of energies, we come to the algebraic equation:

$$
1 \simeq \frac{2 \alpha_{s}}{9 \pi} \int_{\left|\Delta^{(-)}\right|}^{\mu} \frac{d x}{x} \ln \frac{2(2 \mu)^{3}}{\pi M^{2} x} \simeq \frac{\alpha_{s}}{9 \pi} \ln ^{2} \frac{\Lambda}{\left|\Delta^{(-)}\right|},
$$

leading to the following estimate for the gap:

$$
\left|\Delta^{(-)}\right| \simeq \frac{(4 \pi)^{3 / 2} \mu}{\alpha^{5 / 2}} e^{3 d / 2} \exp \left(-3 \sqrt{\frac{\pi}{\alpha_{s}}}\right) .
$$

A more rigorous solution of the integral equation (39) is presented in Appendix B. The latter gives a slightly smaller value of the gap:

$$
\left|\Delta^{(-)}\right| \simeq \frac{(4 \pi)^{3 / 2} \mathrm{e} \mu}{\alpha^{5 / 2}} e^{3 d / 2} \exp \left(-\frac{3 \pi^{3 / 2}}{2^{3 / 2} \sqrt{\alpha_{s}}}\right)=\frac{2^{7} \pi^{4} \mathrm{e} \mu}{g_{s}^{5}} e^{3 d / 2} \exp \left(-\frac{3 \pi^{2}}{\sqrt{2} g_{s}}\right),
$$

where $\mathrm{e}=2.718 \ldots$. Notice that the exponent in this expression is completely determined by the magnetic modes. The main role of the electric modes is in changing the power of $g_{s}$ in the prefactor: from $g_{s}^{-2}$ to $g_{s}^{-5}$. At last, the contribution of the longitudinal modes in the prefactor is formally of order one and subleading, though it is essentially different from 1 for large values of the gauge parameter $|d|$. Obviously, a proper consideration of all subleading effects (vertex corrections, wave function renormalizations, the Meissner effect, etc.) would result in the cancellation of the gauge dependence in the order parameter, though it is hard to show this explicitly. Our consideration here just indicates that subleading corrections in the prefactor might be, though of order one, not small.

The exponent and the power of $g_{s}$ in the prefactor in expression (42) coincide with those in the expression for the gap obtained in Ref. [20] by using the renormalization group method. Also, up to an overall factor of order one, this expression agrees with those in recent papers Refs. [32.33] where the SD equation in dense QCD has been studied by using somewhat different approaches.

Regarding the validity of the expression in Eq. (42), one has to bare in mind that most of the approximations made at the intermediate steps are adequate only for small values of the coupling constant. Therefore, in the most interesting case of the values of $\alpha_{s} \sim O(1)$, the estimate in Eq. (42) is not very reliable. Still we believe that it should give a correct order of magnitude for $\left|\Delta^{(-)}\right|$.

\section{CONCLUSION}

In this paper, we studied the phenomenon of color superconductivity in dense QCD with two light flavors in the framework of the SD equation in the improved rainbow approximation. Since our approach is based on the SD equations with a perturbative kernel, it may provide a reliable description of the system only at sufficiently large values of the chemical potential (say, larger than about a few $\mathrm{GeV}$ ). Remarkably, the latter restriction on the range of validity is also sufficient for suppressing the non-perturbative contributions given by the instanton effects [9].

Regarding our analytical dependence of the gap on the coupling constant presented in Eq. (42), a few comments are in order. First of all, because of the large prefactor in Eq. (42), the magnitude of the gap for realistic values of the chemical potential (200 to $700 \mathrm{MeV}$ ) and the coupling constant (0.8 to 0.4 , respectively) can be as large as of order $100 \mathrm{MeV}$ (see Fig. 11). Therefore, it is of the same order as the non-perturbative contribution due to the instantons. 
When the quark density is sufficiently large, however, the instanton effects become negligible and the order parameter is primarily determined by the gluon interaction. Indeed, the key point to notice is that the expression in Eq. (42) contains $\alpha_{s}^{-1 / 2}$ in the exponent in contrast to $\alpha_{s}^{-1}$ as appears in models with local four-fermion interactions induced, for example, by screened gluons or by instantons. Therefore, at small values of $\alpha_{s}$, the estimate in Eq. (42) is much larger compared to the estimates from four-fermion models.

Thus, our analysis clearly shows that the long-range interaction, mediated by the unscreened magnetic modes of gluons, is responsible for the enhancement of the superconducting gap in the asymptotic region of high densities. This confirms the general conclusions of Ref. [20] based primarily on renormalization group methods.

It is natural to conjecture that expression (42), derived in the improved rainbow approximation, yields the exact essential singularity for the gap at $\alpha_{s}(\mu)=0$ (corresponding to the chemical potential $\mu$ going to infinity). In other words, the improved rainbow approximation can be a good leading approximation for large values of the chemical potential when the instanton contributions are suppressed. Indeed, the relevant region of momenta in the gap equation (38) is $\left|\Delta^{(-)}\right| \ll\left|q_{4}\right| \ll \mu$. Since $\alpha_{s}(\mu) \sim 1 / \ln \left(\mu / \Lambda_{Q C D}\right)$, expression (42) implies that the gap $\left|\Delta^{(-)}\right|$goes to infinity with $\mu$. Therefore the running coupling is small in that relevant region. This suggests that the exponent in expression (42) for the gap is exact (on the other hand, the prefactor can be influenced by higher order corrections in the kernel). This issue deserves further study.

We note that the two approaches, i.e., using either the perturbative interaction or the non-perturbative instanton mediated one, seem to be complimentary ways of describing the intermediate region of dense QCD. While the standard perturbative approach works well at large chemical potential, the instanton approach gives a more appropriate description at small chemical potential.

\section{ACKNOWLEDGMENTS}

We thank R. Pisarski for discussions having clarified some inconsistencies in the original version of the paper. I.A.S. would like to thank the hospitality of Aspen Center for Physics where a part of this work has been done. He also acknowledges useful discussions with W. Bardeen, P. Damgaard, F. P. Esposito, E. Gorbar and M. Jarrell. One of us (D.K.H.) thanks S. Hsu for useful discussions and wishes to acknowledge the financial support of the Korea Research Foundation made in the program year of 1998 (1998-15-D00022). The work of I.A.S. and L.C.R.W. was supported by the U.S. Department of Energy Grant No. DE-FG02-84ER40153.

\section{APPENDIX A: FORMULAE USED IN DERIVATION OF THE GAP EQUATION}

In derivation of Eqs. (32) - (34), we find it convenient to use the following representations for the kinetic terms of the quark propagators:

$$
\begin{aligned}
\left(p_{0}+\mu\right) \gamma^{0}+B \vec{p} & =\gamma^{0}\left[\left(p_{0}-B|\vec{p}|+\mu\right) \Lambda_{p}^{(+)}+\left(p_{0}+B|\vec{p}|+\mu\right) \Lambda_{p}^{(-)}\right], \\
\left(p_{0}-\mu\right) \gamma^{0}+B \vec{p} & =\gamma^{0}\left[\left(p_{0}-B|\vec{p}|-\mu\right) \Lambda_{p}^{(+)}+\left(p_{0}+B|\vec{p}|-\mu\right) \Lambda_{p}^{(-)}\right], \\
{\left[\left(p_{0}+\mu\right) \gamma^{0}+B \vec{p}\right]^{-1} } & =\gamma^{0}\left[\frac{1}{p_{0}+B|\vec{p}|+\mu} \Lambda_{p}^{(+)}+\frac{1}{p_{0}-B|\vec{p}|+\mu} \Lambda_{p}^{(-)}\right], \\
{\left[\left(p_{0}-\mu\right) \gamma^{0}+B \vec{p}\right]^{-1} } & =\gamma^{0}\left[\frac{1}{p_{0}+B|\vec{p}|-\mu} \Lambda_{p}^{(+)}+\frac{1}{p_{0}-B|\vec{p}|-\mu} \Lambda_{p}^{(-)}\right],
\end{aligned}
$$

where the projectors $\Lambda_{p}^{( \pm)}$are defined in Eq. (35) in the main text.

In order to perform the angular integration in the right hand sides of the gap equation (32), one first need to calculate the following two types of traces over the Dirac indices:

$$
\begin{aligned}
& \operatorname{tr}\left[\Lambda_{p}^{( \pm)} \gamma^{\mu} \Lambda_{q}^{( \pm)} \gamma^{\nu}\right]=g^{\mu \nu}(1+t)-2 g^{\mu 0} g^{\nu 0} t+\frac{\vec{q}^{\mu} \vec{p}^{\nu}+\vec{q}^{\nu} \vec{p}^{\mu}}{|\vec{q}||\vec{p}|}+\ldots \\
& \operatorname{tr}\left[\Lambda_{p}^{( \pm)} \gamma^{\mu} \Lambda_{q}^{(\mp)} \gamma^{\nu}\right]=g^{\mu \nu}(1-t)+2 g^{\mu 0} g^{\nu 0} t-\frac{\vec{q}^{\mu} \vec{p}^{\nu}+\vec{q}^{\nu} \vec{p}^{\mu}}{|\vec{q}||\vec{p}|}+\ldots,
\end{aligned}
$$

where $t=\cos \theta$ is the cosine of the angle between three-vectors $\vec{q}$ and $\vec{p}$, and irrelevant antisymmetric terms are denoted by ellipsis. 
By contracting these traces with the projectors of the magnetic, electric and longitudinal types of gluon modes, we arrive at

$$
\begin{aligned}
& O_{\mu \nu}^{(1)} \operatorname{tr}\left[\Lambda_{p}^{( \pm)} \gamma^{\mu} \Lambda_{q}^{( \pm)} \gamma^{\nu}\right]=2(1+t) \frac{q^{2}+p^{2}-q p(1+t)}{q^{2}+p^{2}-2 q p t} \\
& O_{\mu \nu}^{(1)} \operatorname{tr}\left[\Lambda_{p}^{( \pm)} \gamma^{\mu} \Lambda_{q}^{(\mp)} \gamma^{\nu}\right]=2(1-t) \frac{q^{2}+p^{2}+q p(1-t)}{q^{2}+p^{2}-2 q p t}, \\
& O_{\mu \nu}^{(2)} \operatorname{tr}\left[\Lambda_{p}^{( \pm)} \gamma^{\mu} \Lambda_{q}^{( \pm)} \gamma^{\nu}\right]=2(1-t) \frac{q^{2}+p^{2}+q p(1-t)}{q^{2}+p^{2}-2 q p t}-(1-t) \frac{(q+p)^{2}+\left(q_{4}-p_{4}\right)^{2}}{q^{2}+p^{2}-2 q p t+\left(q_{4}-p_{4}\right)^{2}}, \\
& O_{\mu \nu}^{(2)} \operatorname{tr}\left[\Lambda_{p}^{( \pm)} \gamma^{\mu} \Lambda_{q}^{(\mp)} \gamma^{\nu}\right]=2(1+t) \frac{q^{2}+p^{2}-q p(1+t)}{q^{2}+p^{2}-2 q p t}-(1+t) \frac{(q-p)^{2}+\left(q_{4}-p_{4}\right)^{2}}{q^{2}+p^{2}-2 q p t+\left(q_{4}-p_{4}\right)^{2}}, \\
& O_{\mu \nu}^{(3)} \operatorname{tr}\left[\Lambda_{p}^{( \pm)} \gamma^{\mu} \Lambda_{q}^{( \pm)} \gamma^{\nu}\right]=(1-t) \frac{(q+p)^{2}+\left(q_{4}-p_{4}\right)^{2}}{q^{2}+p^{2}-2 q p t+\left(q_{4}-p_{4}\right)^{2}}, \\
& O_{\mu \nu}^{(3)} \operatorname{tr}\left[\Lambda_{p}^{( \pm)} \gamma^{\mu} \Lambda_{q}^{(\mp)} \gamma^{\nu}\right]=(1+t) \frac{(q-p)^{2}+\left(q_{4}-p_{4}\right)^{2}}{q^{2}+p^{2}-2 q p t+\left(q_{4}-p_{4}\right)^{2}},
\end{aligned}
$$

where $q \equiv|\vec{q}|, p \equiv|\vec{p}|, q_{4} \equiv-i q_{0}$ and $p_{4} \equiv-i p_{0}$.

Now, by making use of the explicit expressions (A7), we easily perform the angular integrations of all types appearing in the gap equations:

$$
\begin{aligned}
& \int \frac{d \Omega|\vec{q}-\vec{p}|}{|\vec{q}-\vec{p}|^{3}+\omega_{l}^{3}} O_{\mu \nu}^{(1)} \operatorname{tr}\left[\Lambda_{p}^{( \pm)} \gamma^{\mu} \Lambda_{q}^{( \pm)} \gamma^{\nu}\right]=\pi\left[-\frac{2}{q p}+\frac{\left(q^{2}-p^{2}\right)^{2}+\omega_{l}^{4}}{\sqrt{3} \omega_{l}^{2} q^{2} p^{2}} \arctan \left(\frac{\sqrt{3} \omega_{l} \min (q, p)}{\omega_{l}^{2}+\left|q^{2}-p^{2}\right|-\omega_{l} \max (q, p)}\right)\right. \\
& \left.+\frac{\left(q^{2}-p^{2}\right)^{2}-\omega_{l}^{4}}{3 \omega_{l}^{2} q^{2} p^{2}} \ln \frac{\omega_{l}+|q+p|}{\omega_{l}+|q-p|}-\frac{\left(q^{2}-p^{2}\right)^{2}-\omega_{l}^{4}}{6 \omega_{l}^{2} q^{2} p^{2}} \ln \frac{\omega_{l}^{2}+|q+p|^{2}-\omega_{l}|q+p|}{\omega_{l}^{2}+|q-p|^{2}-\omega_{l}|q-p|}+\frac{4}{3 q p} \ln \frac{\omega_{l}^{3}+|q+p|^{3}}{\omega_{l}^{3}+|q-p|^{3}}\right],
\end{aligned}
$$

where $\omega_{l}^{3}=(\pi / 2) M^{2} \omega$ and $\omega=\left|q_{4}-p_{4}\right|$. Similarly,

$$
\begin{gathered}
\int \frac{d \Omega|\vec{q}-\vec{p}|}{|\vec{q}-\vec{p}|^{3}+\omega_{l}^{3}} O_{\mu \nu}^{(1)} \operatorname{tr}\left[\Lambda_{p}^{( \pm)} \gamma^{\mu} \Lambda_{q}^{(\mp)} \gamma^{\nu}\right]=\pi\left[\frac{2}{q p}-\frac{\left(q^{2}-p^{2}\right)^{2}+\omega_{l}^{4}}{\sqrt{3} \omega_{l}^{2} q^{2} p^{2}} \arctan \left(\frac{\sqrt{3} \omega_{l} \min (q, p)}{\omega_{l}^{2}+\left|q^{2}-p^{2}\right|-\omega_{l} \max (q, p)}\right)\right. \\
\left.-\frac{\left(q^{2}-p^{2}\right)^{2}-\omega_{l}^{4}}{3 \omega_{l}^{2} q^{2} p^{2}} \ln \frac{\omega_{l}+|q+p|}{\omega_{l}+|q-p|}+\frac{\left(q^{2}-p^{2}\right)^{2}-\omega_{l}^{4}}{6 \omega_{l}^{2} q^{2} p^{2}} \ln \frac{\omega_{l}^{2}+|q+p|^{2}-\omega_{l}|q+p|}{\omega_{l}^{2}+|q-p|^{2}-\omega_{l}|q-p|}+\frac{4}{3 q p} \ln \frac{\omega_{l}^{3}+|q+p|^{3}}{\omega_{l}^{3}+|q-p|^{3}}\right] \\
\quad \int \frac{d \Omega}{|\vec{q}-\vec{p}|^{2}+\omega^{2}+2 M^{2}} O_{\mu \nu}^{(2)} \operatorname{tr}\left[\Lambda_{p}^{( \pm)} \gamma^{\mu} \Lambda_{q}^{( \pm)} \gamma^{\nu}\right]=\frac{2 \pi}{q p}+\frac{\pi}{2 q^{2} p^{2}}\left[-\frac{\left(q^{2}-p^{2}\right)^{2}}{2 M^{2}+\omega^{2}} \ln \frac{(q+p)^{2}}{(q-p)^{2}}\right. \\
-\frac{\left[(q-p)^{2}+2 M^{2}+\omega^{2}\right]\left[\left(2 M^{2}+\omega^{2}\right)^{2}+(q+p)^{2} \omega^{2}\right]}{2 M^{2}\left(2 M^{2}+\omega^{2}\right)} \ln \frac{(q+p)^{2}+2 M^{2}+\omega^{2}}{(q-p)^{2}+2 M^{2}+\omega^{2}} \\
\left.+\frac{\left[(q+p)^{2}+\omega^{2}\right]\left[(q-p)^{2}+\omega^{2}\right]}{2 M^{2}} \ln \frac{(q+p)^{2}+\omega^{2}}{(q-p)^{2}+\omega^{2}}\right], \\
\quad \int \frac{d \Omega}{|\vec{q}-\vec{p}|^{2}+\omega^{2}+2 M^{2}} O_{\mu \nu}^{(2)} \operatorname{tr}\left[\Lambda_{p}^{( \pm)} \gamma^{\mu} \Lambda_{q}^{(\mp)} \gamma^{\nu}\right]=-\frac{2 \pi}{q p}+\frac{\pi}{2 q^{2} p^{2}}\left[\frac{\left(q^{2}-p^{2}\right)^{2}}{2 M^{2}+\omega^{2}} \ln \frac{(q+p)^{2}}{(q-p)^{2}}\right. \\
+\frac{\left[(q+p)^{2}+2 M^{2}+\omega^{2}\right]\left[\left(2 M^{2}+\omega^{2}\right)^{2}+(q-p)^{2} \omega^{2}\right]}{2 M^{2}\left(2 M^{2}+\omega^{2}\right)} \ln \frac{(q+p)^{2}+2 M^{2}+\omega^{2}}{(q-p)^{2}+2 M^{2}+\omega^{2}} \\
\left.-\frac{\left[(q+p)^{2}+\omega^{2}\right]\left[(q-p)^{2}+\omega^{2}\right]}{2 M^{2}} \ln \frac{(q+p)^{2}+\omega^{2}}{(q-p)^{2}+\omega^{2}}\right], \\
d \int \frac{d \Omega}{|\vec{q}-\vec{p}|^{2}+\omega^{2}} O_{\mu \nu}^{(3)} \operatorname{tr}\left[\Lambda_{p}^{( \pm)} \gamma^{\mu} \Lambda_{q}^{( \pm)} \gamma^{\nu}\right]=\pi d\left[-\frac{2}{q p}+\frac{(q+p)^{2}+\omega^{2}}{2 q^{2} p^{2}} \ln \frac{(q+p)^{2}+\omega^{2}}{(q-p)^{2}+\omega^{2}}\right],
\end{gathered}
$$




$$
d \int \frac{d \Omega}{|\vec{q}-\vec{p}|^{2}+\omega^{2}} O_{\mu \nu}^{(3)} \operatorname{tr}\left[\Lambda_{p}^{( \pm)} \gamma^{\mu} \Lambda_{q}^{(\mp)} \gamma^{\nu}\right]=\pi d\left[\frac{2}{q p}-\frac{(q-p)^{2}+\omega^{2}}{2 q^{2} p^{2}} \ln \frac{(q+p)^{2}+\omega^{2}}{(q-p)^{2}+\omega^{2}}\right]
$$

As a result, the angular average of the gluon propagator (multiplied by $q^{2}$ weight) in the vicinity of the Fermi surface is given by the following approximate expression:

$$
q^{2} \int d \Omega \mathcal{D}_{\mu \nu}(q-p) \operatorname{tr}\left[\Lambda_{p}^{( \pm)} \gamma^{\mu} \Lambda_{q}^{(\mp)} \gamma^{\nu}\right] \approx 2 i \pi\left[\frac{2}{3} \ln \frac{(2 \mu)^{3}}{\left|\epsilon_{q}^{-}\right|^{3}+\omega_{l}^{3}}+\ln \frac{(2 \mu)^{2}}{\left(\epsilon_{q}^{-}\right)^{2}+2 M^{2}+\omega^{2}}+d\right]
$$

where $\epsilon_{q}^{-}=q-\mu$ and $d$ if the gauge fixing parameter. The three terms in the last expression are the leading contributions of the magnetic, electric and the longitudinal gluon modes, respectively. Because of the absence of the logarithmic factor in front of the gauge fixing parameter, the longitudinal gluon modes become relevant only in the next-to-leading order.

Now, after performing the approximate integration over the spatial momentum, we arrive at

$$
\begin{aligned}
& \int_{0}^{\mu} \frac{q^{2} d q}{q_{4}^{2}+\left(\epsilon_{q}^{-}\right)^{2}+|\Delta|^{2}} \int d \Omega \mathcal{D}_{\mu \nu}(q-p) \operatorname{tr}\left[\Lambda_{p}^{( \pm)} \gamma^{\mu} \Lambda_{q}^{(\mp)} \gamma^{\nu}\right] \\
\approx & \frac{4 i \pi^{2}}{3 \sqrt{q_{4}^{2}+|\Delta|^{2}}}\left(\ln \frac{(2 \mu)^{3}}{\omega_{l}^{3}}+\frac{3}{2} \ln \frac{(2 \mu)^{2}}{2 M^{2}+\omega^{2}}+\frac{3}{2} d\right) \approx \frac{4 i \pi^{2}}{3 \sqrt{q_{4}^{2}+|\Delta|^{2}}} \ln \frac{\Lambda}{\omega},
\end{aligned}
$$

where $\Lambda \equiv e^{3 d / 2}(2 \mu)^{6} /\left(\sqrt{2} \pi M^{5}\right)=e^{3 d / 2} 16(2 \pi)^{3 / 2} \mu /\left(N_{f} \alpha_{s}\right)^{5 / 2}$.

\section{APPENDIX B: THE SOLUTION OF THE GAP EQUATION}

In this Appendix we present a somewhat more rigorous solution of the integral equation (39).

In order to rewrite it in the form of a differential equation, we approximate the logarithm in the kernel by its asymptotes (below $\Delta \equiv \Delta^{(-)}, p \equiv p_{4}$ ):

$$
\Delta(p) \simeq \frac{2 \alpha_{s}}{9 \pi} \int_{0}^{p} \frac{d q \Delta(q)}{\sqrt{q^{2}+|\Delta|^{2}}} \ln \frac{\Lambda}{p}+\frac{2 \alpha_{s}}{9 \pi} \int_{p}^{\Lambda} \frac{d q \Delta(q)}{\sqrt{q^{2}+|\Delta|^{2}}} \ln \frac{\Lambda}{q},
$$

where, without loss of generality, $\Lambda=e^{3 d / 2}(4 \pi)^{3 / 2} \mu / \alpha^{5 / 2}$, appearing in the logarithm, is also used as an ultraviolet cut-off in integration over $q$. This equation, as is easy to check, is equivalent to the differential equation:

$$
p \Delta^{\prime \prime}(p)+\Delta^{\prime}(p)+\frac{2 \alpha_{s}}{9 \pi} \frac{\Delta(p)}{\sqrt{p^{2}+|\Delta|^{2}}}=0,
$$

along with the following infrared and ultraviolet boundary conditions:

$$
\left.p \Delta^{\prime}(p)\right|_{p=0}=0 \quad(\mathrm{IR}), \quad \Delta(\Lambda)=0 \quad(\mathrm{UV}) .
$$

We solve the differential equation (B2 analytically in two regions $p \ll|\Delta|$ and $p \gg|\Delta|$ and then match the solutions at $p=|\Delta|$.

In the infrared region $p \ll|\Delta|$, the solution that satisfies the IR boundary condition is given by the following Bessel function:

$$
\Delta(p)=|\Delta| J_{0}\left(\nu \sqrt{\frac{p}{|\Delta|}}\right)
$$

where, by definition, $\nu=\sqrt{8 \alpha_{s} / 9 \pi}$ and the overall constant was chosen in such a way that $\Delta(0)=|\Delta|$.

In the other region, $p \gg|\Delta|$, the solution, consistent with the UV boundary condition, reads

$$
\Delta(p)=B \sin \left(\frac{\nu}{2} \ln \frac{\Lambda}{p}\right) .
$$

While matching the solutions and their derivatives at the point $p=|\Delta|$, we get the relation: 


$$
|\Delta|=\Lambda \exp \left[-\frac{2}{\nu} \arctan \left(\frac{J_{0}(\nu)}{J_{1}(\nu)}\right)\right]
$$

and determine the value of the constant $B$ :

$$
B=|\Delta| \sqrt{J_{0}^{2}(\nu)+J_{1}^{2}(\nu)}
$$

The dependence ( $\mathrm{B} 6)$ of the order parameter on the chemical potential is presented in Fig. 1. Here, to establish the function $\alpha_{s}(\mu)$, we fix the magnitude of the QCD coupling constant by choosing $\alpha_{s}(1.76 \mathrm{GeV}) \approx 0.26$. Then, by making use of the one-loop running of $\alpha_{s}$, we obtain the functional dependence of $\alpha_{s}$ on $\mu$.

Finally, assuming that the coupling constant is small, $\nu \ll 1$, we arrive at the analytical expression presented in Eq. (42):

$$
|\Delta| \simeq \Lambda \exp \left(-\frac{\pi}{\nu}+1+O\left(\nu^{2}\right)\right)
$$

[1] D.J. Gross and F. Wilczek, Phys. Rev. Lett. 30, 1343 (1973); H.D. Politzer, Phys. Rev. Lett. 30, 1346 (1973).

[2] M.A. Shifman, A.I. Vainshtein and V.I. Zakharov, Nucl. Phys. B147, 385, (1979); ibid. B147, 448 (1979).

[3] J.B. Kogut, Rev. Mod. Phys. 55, 775 (1983).

[4] T. Schaefer and E.V. Shuryak, Rev. Mod. Phys. 70, 323 (1998).

[5] P.I. Fomin, V.P. Gusynin, V.A. Miransky, and Yu.A. Sitenko, Riv. Nuovo Cimento 6, 1 (1983); V.A. Miransky, Dynamical Symmetry Breaking in Quantum field Theories (World Scientific, Singapore, 1993); C.D. Roberts and A.G. Williams, Prog. Part. Nucl. Phys. 33, 477 (1994).

[6] D. Bailin and A. Love, Nucl. Phys. B190, 175 (1981); Nucl. Phys. B205, 119 (1982); Phys. Rep. 107, 325 (1984).

[7] M. Alford, K. Rajagopal and F. Wilczek, Phys. Lett. B422, 247 (1998).

[8] R. Rapp, T. Schaefer, E.V. Shuryak and M. Velkovsky, Phys. Rev. Lett. 81, 53 (1998).

[9] R. Rapp, T. Schaefer, E.V. Shuryak and M. Velkovsky, hep-ph/9904353.

[10] S. Hands and S.E. Morrison, Phys. Rev. D 59 (1999) 116002.

[11] E. Witten, Phys. Rev. D 30, 272 (1984).

[12] M. Alford, K. Rajagopal and F. Wilczek, Nucl. Phys. B537, 443 (1999).

[13] J. Berges and K. Rajagopal, Nucl. Phys. B538, 215 (1999).

[14] M. Alford, J. Berges and K. Rajagopal, hep-ph/9903502.

[15] T. Schaefer and F. Wilczek, hep-ph/9903503.

[16] G.W. Carter and D. Diakonov, Nucl. Phys. A642, 78 (1998); Phys. Rev. D 60, 016004 (1999).

[17] K. Langfeld and M. Rho, hep-ph/9811227.

[18] N. Evans, S.D.H. Hsu and M. Schwetz, Nucl. Phys. B551, 275 (1999); Phys. Lett. B449, 281 (1999); S.D.H. Hsu, nuclth/9903039.

[19] R.D. Pisarski and D.H. Rischke, Phys. Rev. Lett. 83, 37 (1999).

[20] D.T. Son, Phys. Rev. D 59, 094019 (1999).

[21] D.K. Hong, hep-ph/9812510; hep-ph/9905523.

[22] N.O. Agasian, B.O. Kerbikov, and V.I. Shevchenko, hep-ph/9902335.

[23] J.C. Collins and M.J. Perry, Phys. Rev. Lett. 34, 1353 (1975).

[24] M. Iwasaki and T. Iwado, Phys. Lett. B 350, 163 (1995).

[25] H. Vija and M.H. Thoma, Phys. Lett. B 342, 212 (1995).

[26] U. Heinz, Ann. Phys. (NY) 168, 148 (1986).

[27] C. Manuel, Phys. Rev. D 53, 5866 (1996); G. Alexanian and V.P. Nair, Phys. Lett. B390, 370 (1997).

[28] O.K. Kalashnikov and V.V. Klimov, Sov. J. Nucl. Phys. 31, 699 (1980).

[29] M. Le Bellac and C. Manuel, Phys. Rev. D 55, 3215 (1997); B. Vanderheyden and J.-Y. Ollitrault, ibid. 56, 5108 (1997).

[30] E.M. Lifshitz and L.P. Pitaevskii, Statistical Physics, Part 2 (Pergamon Press, Oxford, 1980).

[31] A.L. Fetter and J.D. Walecka, Quantum Theory of Many-Particle Systems (McGraw-Hill, New York, 1971).

[32] T. Schaefer and F. Wilczek, hep-ph/9906512.

[33] R.D. Pisarski and D.H. Rischke, nucl-th/9907041. 


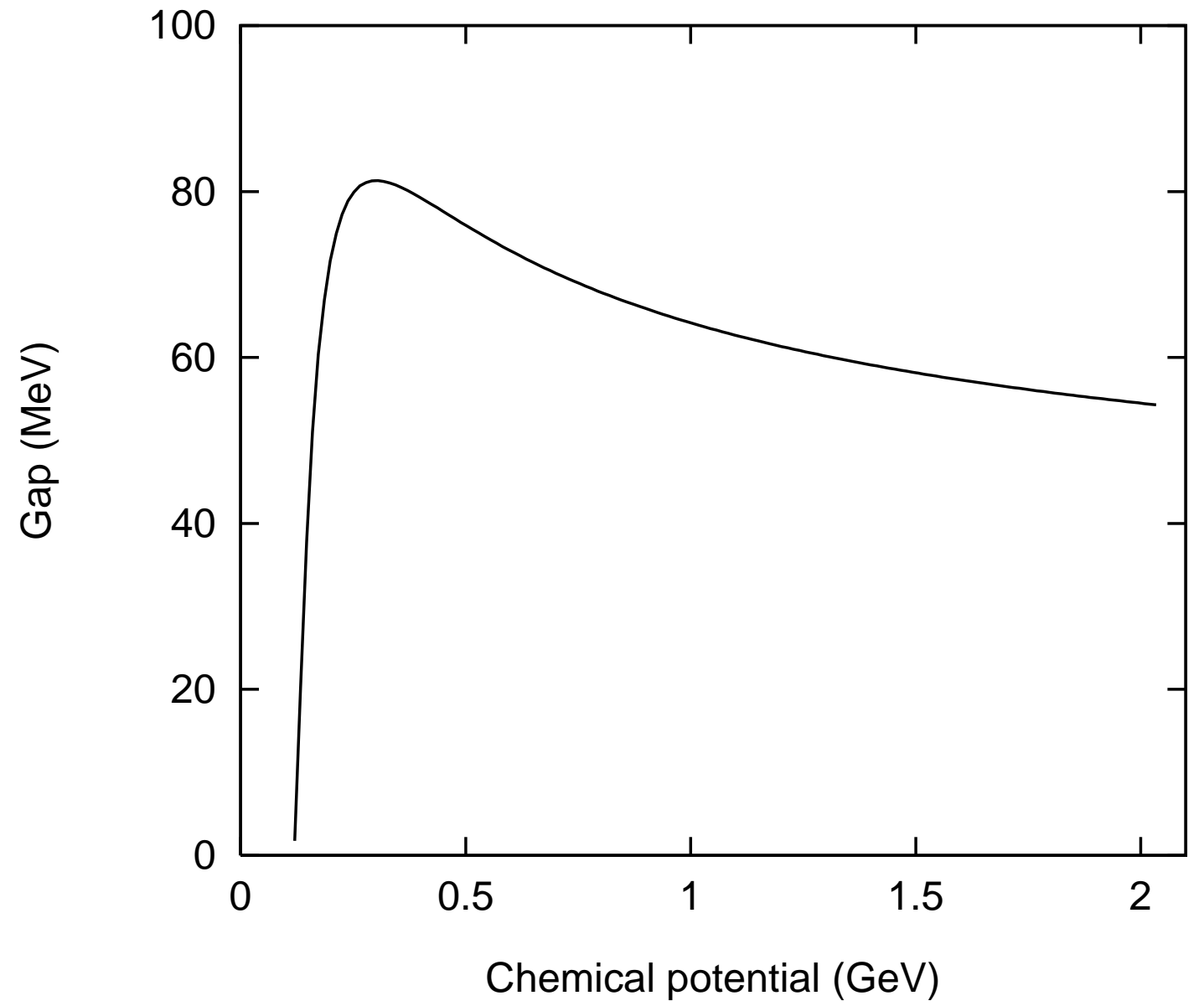

FIG. 1. The dependence of the order parameter on the chemical potential. The function $\alpha_{s}(\mu)$ is established by fixing $\alpha_{s}(1.76 \mathrm{GeV}) \approx 0.26$ and using the one-loop running of the coupling constant. 